\title{
Optimum Switching Pattern of Matrix Converter Space Vector Modulation
}

\author{
Amir Masoud Bozorgi, Mohammad Monfared, and Habib Rajabi Mashhadi \\ Department of Electrical Engineering, \\ Ferdowsi University of Mashhad, \\ Mashhad, Iran. \\ amir.bozorgi@ieee.org,m.monfared@um.ac.ir, and h_mashhadi@um.ac.ir
}

\begin{abstract}
This paper presents an optimum switching pattern for the space vector modulation (SVM) of matrix converters based on a genetic algorithm. The possibility of choosing the sequence of active duty cycles, and the ability to allocate the duration of the inactive duty cycle to each one of the zero duty cycles in a matrix converter SVM, provide some degrees of freedom in designing of the switching pattern. This leads to an optimization problem. Therefore, a suitable genetic algorithm for solving the switching pattern optimization problem is adopted, and an objective function to minimize the weighted total harmonic distortion (WTHD) and the low-order harmonics of the output voltage is proposed. Simulations in Matlab/Simulink confirm the validity of analytical achievements.

Keywords- Matrix converter; space vector modulation (SVM); switching pattern; gentic algorithms (GA)
\end{abstract}

\section{INTRODUCTION}

The rapid growth of Ac-Ac converters in industrial applications on one side, and an inclination to reduce the total cost of converters have led to a great interest in matrix converters [1]. As a total silicon converter, a matrix converter eliminates the need for dc-link capacitor in comparison with AC-DC-AC converters. Fig. 1 depicts the topology of a 3-phase to 3-phase matrix converter.

Several modulation strategies have been reported for matrix converters [2]. Due to the bidirectional power flow capability, almost sinusoidal input/output waveforms, the ability to control the output voltage vector (magnitude and phase angle), and the adjustable input power factor, the space vector modulation (SVM) has been known as one of the most popular modulation techniques for matrix converters [3], [4].

The matrix converter SVM offers several degrees of freedom in designing an appropriate switching pattern. The designed switching pattern can follow many targets, among them minimizing the switching numbers and the harmonic contents of output voltage are the most important [5]. In 1995, the 8 switch method (8 commutations per switching period) was presented in [6], which results in the minimum switching numbers in a period by using only one zero vector in conjunction with four active vectors. In [7], an approach to reduce the distortion of output voltage was proposed which

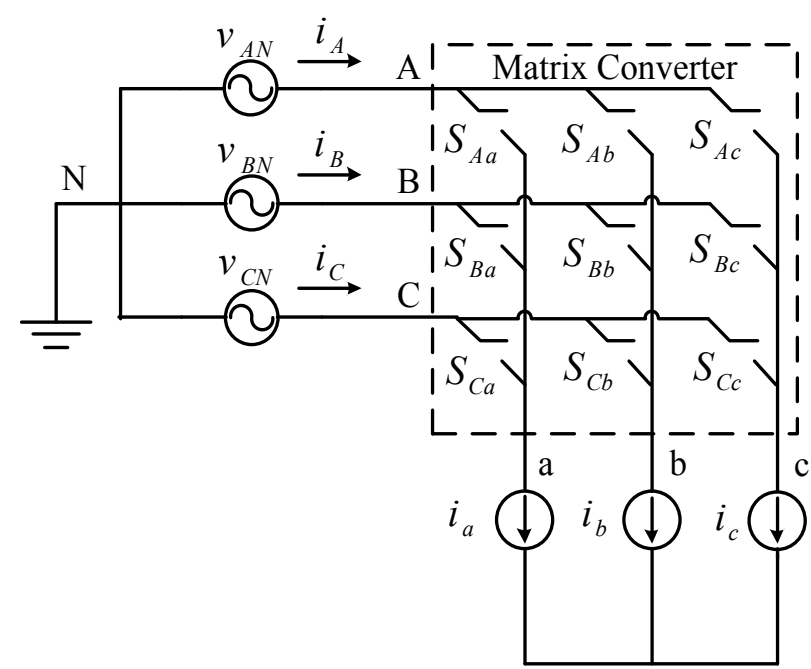

n

Figure 1. The structure of a 3-phase to 3-phase matrix converter.

utilizes another zero vector at the end of switching sequence leading to 10 commutations per switching period. Another successful work has the low switching numbers (8 commutations), and at the same time provides a low harmonic distortion [5]. Some other works have focused on other goals rather than minimum commutations or reduced harmonic distortions [8], [9], [10].

In this paper, a method to design the switching pattern of the SVM matrix converter based on a genetic algorithm is presented. An objective function to minimize the THD of output voltage is proposed. The simulation results confirm the performance of the proposed method.

\section{Direct Space Vector Modulation}

This modulation is based on transforming the input and output quantities into space vectors. The space vector of three-phase quantities can be obtained through the following transformation

$$
\bar{X}=\frac{2}{3}\left(x_{a}+\overline{a x} x_{b}+\bar{a}^{2} x_{c}\right)
$$

where $x_{a}, x_{b}$ and $x_{c}$ are the three-phase quantities, and $\bar{a}$ is a complex constant defined as follows

$$
\bar{a}=e^{j \frac{2 \pi}{3}}=\cos \left(\frac{2 \pi}{3}\right)+j \sin \left(\frac{2 \pi}{3}\right) .
$$


Therefore, if the output voltages and input currents are assumed balanced and sinusoidal, their space vectors can be readily expressed as

$$
\begin{gathered}
\bar{V}_{\text {out }}=\frac{2}{3}\left(v_{a}+v_{b} e^{j \frac{2 \pi}{3}}+v_{c} e^{j \frac{4 \pi}{3}}\right)=V_{\text {om }} e^{j \omega_{o} t-\varphi_{o}} \\
\bar{I}_{\text {in }}=\frac{2}{3}\left(i_{A}+i_{B} e^{j \frac{2 \pi}{3}}+i_{C} e^{j \frac{4 \pi}{3}}\right)=I_{\text {im }} e^{j \omega_{i} t-\varphi_{i}}
\end{gathered}
$$

where $\omega_{o}$, and $\omega_{i}$ are the output and input angular frequencies, respectively, and $\varphi_{o}$, and $\varphi_{i}$ are the initial phase angles of the output voltage and input current vectors, respectively. Furthermore, the amplitude of the output voltage and input current are indicated with $V_{o m}$ and $I_{i m}$, respectively.

On the other hand, given that the switching states leading to input voltage short circuit or output terminals open circuit must be avoided, only 27 possible switching states are possible for the topology of Fig. 1. Among these 27 possible states, only 21 states which lead to stationary vectors will be used for the SVM. Now, if for each switching state, the corresponding output voltages and input currents are substituted in (3) and (4), respectively, their space vectors will be achieved as summarized in table I. Fig. 2 illustrates these vectors.

Assuming $\bar{V}_{\text {out }}$ and $\bar{I}_{\text {in }}$ are in sector 1 and 3, respectively, as shown in Fig. 3, then the reference vectors can be synthesized with resolving them into adjacent active space vectors. Given that both output voltage vector and input current vector should be synthesized at the same time, only the common switching states between the output voltage and input current vectors can be used. As a result, among adjacent active states, only $( \pm 1, \pm 2, \pm 7, \pm 8)$ switching

\begin{tabular}{|c|c|c|c|c|c|c|c|}
\hline \multicolumn{3}{|c|}{ On Switches } & \multirow{2}{*}{$\begin{array}{c}\text { States } \\
+1\end{array}$} & \multirow{2}{*}{$\frac{\left|\overline{v_{o}}\right|}{2 / 3 v_{A B}}$} & \multirow{2}{*}{$\frac{\angle \overline{v_{o}}}{0}$} & \multirow{2}{*}{$\frac{\left|\overline{i_{i n}}\right|}{2 / \sqrt{3} i_{a}}$} & \multirow{2}{*}{$\begin{array}{l}\overline{i_{\text {in }}} \\
-\pi / 6\end{array}$} \\
\hline$S_{A a}$ & $S_{B b}$ & $S_{B c}$ & & & & & \\
\hline$S_{B a}$ & $S_{A b}$ & $S_{A c}$ & -1 & $-2 / 3 v_{A B}$ & 0 & $-2 / \sqrt{ } 3 i_{a}$ & $-\pi / 6$ \\
\hline$S_{B a}$ & $S_{C b}$ & $S_{C c}$ & +2 & $2 / 3 v_{B C}$ & 0 & $2 / \sqrt{ } 3 i_{a}$ & $\pi / 2$ \\
\hline$S_{C a}$ & $S_{B b}$ & $S_{B c}$ & -2 & $-2 / 3 v_{B C}$ & 0 & $-2 / \sqrt{ } 3 i_{a}$ & $\pi / 2$ \\
\hline$S_{C a}$ & $S_{A b}$ & $S_{A c}$ & +3 & $2 / 3 v_{C A}$ & 0 & $2 / \sqrt{ } 3 i_{a}$ & $7 \pi / 6$ \\
\hline$S_{A a}$ & $S_{C b}$ & $S_{C c}$ & -3 & $-2 / 3 v_{C A}$ & 0 & $-2 / \sqrt{ } 3 i_{a}$ & $7 \pi / 6$ \\
\hline$S_{B a}$ & $\overline{S_{A b}}$ & $\overline{S_{B C}}$ & +4 & $2 / 3 v_{A B}$ & $2 \pi / 3$ & $2 / \sqrt{3} i_{b}$ & $-\pi / 6$ \\
\hline$S_{A a}$ & $S_{B b}$ & $S_{A c}$ & -4 & $-2 / 3 v_{A B}$ & $2 \pi / 3$ & $-2 / \sqrt{ } 3 i_{b}$ & $-\pi / 6$ \\
\hline$S_{C a}$ & $S_{B b}$ & $S_{C c}$ & +5 & $2 / 3 v_{B C}$ & $2 \pi / 3$ & $2 / \sqrt{ } 3 i_{b}$ & $\pi / 2$ \\
\hline$S_{B a}$ & $S_{C b}$ & $S_{B c}$ & -5 & $-2 / 3 v_{B C}$ & $2 \pi / 3$ & $-2 / \sqrt{ } 3 i_{b}$ & $\pi / 2$ \\
\hline$S_{A a}$ & $S_{C b}$ & $S_{A c}$ & +6 & $2 / 3 v_{C A}$ & $2 \pi / 3$ & $2 / \sqrt{ } 3 i_{b}$ & $7 \pi / 6$ \\
\hline$S_{C a}$ & $S_{A b}$ & $S_{C c}$ & -6 & $-2 / 3 v_{C A}$ & $2 \pi / 3$ & $-2 / \sqrt{ } 3 i_{b}$ & $7 \pi / 6$ \\
\hline$S_{B a}$ & $S_{B b}$ & $S_{A c}$ & +7 & $2 / 3 v_{A B}$ & $4 \pi / 3$ & $2 / \sqrt{3} i_{c}$ & $-\pi / 6$ \\
\hline$S_{A a}$ & $S_{A b}$ & $S_{B c}$ & -7 & $-2 / 3 v_{A B}$ & $4 \pi / 3$ & $-2 / \sqrt{ } 3 i_{c}$ & $-\pi / 6$ \\
\hline$S_{C a}$ & $S_{C b}$ & $S_{B c}$ & +8 & $2 / 3 v_{B C}$ & $4 \pi / 3$ & $2 / \sqrt{ } 3 i_{c}$ & $\pi / 2$ \\
\hline$S_{B a}$ & $S_{B b}$ & $S_{C c}$ & -8 & $-2 / 3 v_{B C}$ & $4 \pi / 3$ & $-2 / \sqrt{ } 3 i_{c}$ & $\pi / 2$ \\
\hline$S_{A a}$ & $S_{A b}$ & $S_{C c}$ & +9 & $2 / 3 v_{C A}$ & $4 \pi / 3$ & $2 / \sqrt{ } 3 i_{c}$ & $7 \pi / 6$ \\
\hline$S_{C a}$ & $S_{C b}$ & $S_{A c}$ & -9 & $-2 / 3 v_{C A}$ & $4 \pi / 3$ & $-2 / \sqrt{ } 3 i_{c}$ & $7 \pi / 6$ \\
\hline$S_{A a}$ & $S_{A b}$ & $S_{A c}$ & $0_{1}$ & 0 & - & - & 0 \\
\hline$S_{B a}$ & $S_{B b}$ & $S_{B c}$ & $\mathrm{O}_{2}$ & 0 & - & - & 0 \\
\hline$S_{C a}$ & $S_{C b}$ & $S_{C c}$ & $\mathrm{O}_{3}$ & 0 & - & - & 0 \\
\hline
\end{tabular}

TABLE I. POSSIBLE SWITCHING STATES AND THEIR SPACE VECTORS

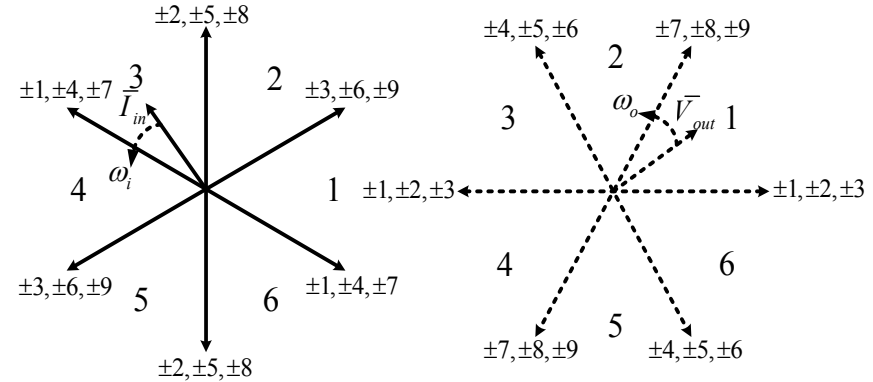

(a)

(b)
Figure 2. Active space vectors of (a) output voltage (b) input current.

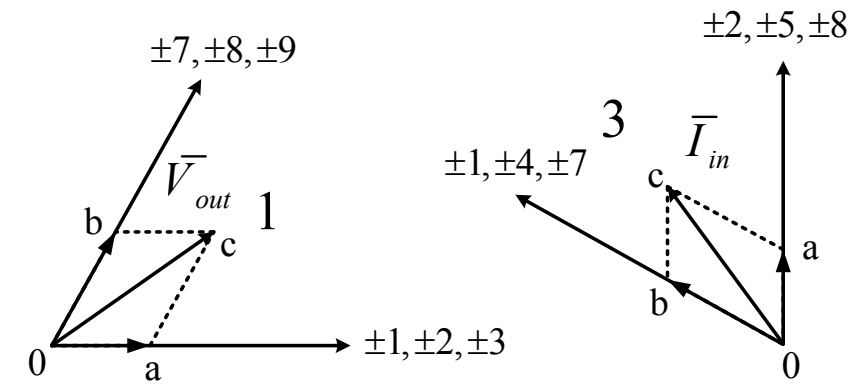

Figure 3. Output voltage space vector in sector 1 and input current space vector in sector 3 .

configurations must be selected. Table II represents the active switching configurations for all possible combinations of output voltage and input current vectors sectors.

In order to obtain the duty cycles of active switching states, reference vectors are written in terms of adjacent vectors [4]:

$$
\begin{gathered}
\bar{V}(1) d_{3}+\bar{V}(2) d_{4}=\bar{V}_{0 a}=\frac{2}{\sqrt{3}} V_{r e f} \sin \left(\tilde{\alpha}_{o}\right) e^{j\left[\left(K_{v}-1\right) \frac{\pi}{3}\right]} \\
\bar{V}(7) d_{1}+\bar{V}(8) d_{2}=\bar{V}_{0 b}=\frac{2}{\sqrt{3}} V_{r e f} \sin \left(\frac{\pi}{3}-\tilde{\alpha}_{o}\right) e^{j\left[\left(K_{v}-1\right) \frac{\pi}{3}+\frac{\pi}{3}\right]} \\
\left(\bar{I}(7) d_{1}+\bar{I}(1) d_{3}\right) \cdot j e^{j \tilde{\beta}_{i}+\pi / 6} e^{j\left[\left(K_{i}-1\right) \frac{\pi}{3}\right]}=0 \\
\left(\bar{I}(2) d_{4}+\bar{I}(8) d_{2}\right) \cdot j e^{j \tilde{\beta}_{i}+\pi / 6} e^{j\left[\left(K_{i}-1\right) \frac{\pi}{3}\right]}=0
\end{gathered}
$$

in which $K_{v}$, and $K_{i}$ are the sectors of the output voltage and input current vectors, respectively, and "." Indicates scalar product, and

$$
\tilde{\alpha}_{o}=\alpha_{o}-\left(K_{v}-1\right) \frac{\pi}{3}, \tilde{\beta}_{i}=\beta_{i}-\left(K_{i}-1\right) \frac{\pi}{3}
$$

where $\alpha_{o}$ and $\beta_{i}$ are the phase angles of the output voltage and input current vectors, respectively. Solving (5) to (8), the duty cycles are obtained as

$$
\begin{gathered}
d_{1}=(-1)^{K_{v}+K_{i}} \frac{2}{\sqrt{3}} q \frac{\sin \left(\tilde{\alpha}_{o}\right) \sin \left(\tilde{\beta}_{i}\right)}{\cos \varphi_{i}} \\
d_{2}=(-1)^{K_{v}+K_{i}+1} \frac{2}{\sqrt{3}} q \frac{\sin \left(\tilde{\alpha}_{o}\right) \sin \left(\frac{\pi}{3}-\tilde{\beta}_{i}\right)}{\cos \varphi_{i}}
\end{gathered}
$$


TABLE II. USED SWITCHING STATES

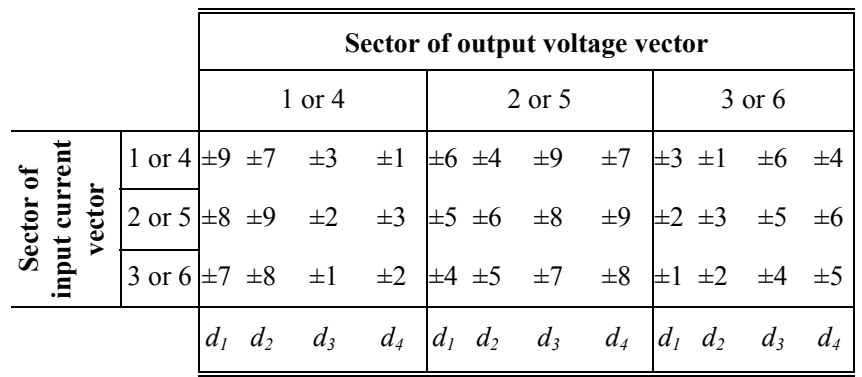

$$
\begin{aligned}
& d_{3}=(-1)^{K_{v}+K_{i}+1} \frac{2}{\sqrt{3}} q \frac{\sin \left(\frac{\pi}{3}-\tilde{\alpha}_{o}\right) \sin \left(\tilde{\beta}_{i}\right)}{\cos \varphi_{i}} \\
& d_{4}=(-1)^{K_{v}+K_{i}} \frac{2}{\sqrt{3}} q \frac{\sin \left(\frac{\pi}{3}-\tilde{\alpha}_{o}\right) \sin \left(\frac{\pi}{3}-\tilde{\beta}_{i}\right)}{\cos \varphi_{i}}
\end{aligned}
$$

where $q$ is the voltage transfer ratio and is defined as the ratio of the output voltage amplitude to the input voltage amplitude.

Considering (10)-(13), it is obvious that two duty cycles are always negative. In the case of these duty cycles, the corresponding negative switching configurations should be chosen instead of the positive ones. Table III represents the input phase voltages connected to the output voltages for the entire possible combinations of the output voltage and input current vectors sectors.

To complete the switching cycle, zero configurations are also added

$$
\sum_{i=1}^{4}\left|d_{i}\right|+\sum_{j=1}^{3} d_{0_{j}}=1 .
$$

in (14), $d_{0 j}$ indicates the duty cycle of $j$-th zero vector in table I.

\section{Application of Genetic Algorithms to Design THE PROPER SWITCHING PATTERN}

\section{A. Potential for an Optimization Problem}

As mentioned earlier, after specifying the active duty cycles, the zero duty cycle will be added to complete the switching period. Choosing one or more zero duty cycles among three available zero duty cycles, and the share of each one in inactive time will be arbitrary. This provides some degrees of freedom in switching pattern design.

In addition, after determining the zero duty cycles, the sequence order of active and zero duty cycles during a switching period can offer another degree of freedom for an optimization problem. Using these features of the SVM switching pattern, some performance characteristics such as the number of switchings and the harmonic contents can be improved.

\section{B. Adoption of Genetic Algorithms for Solving Our Problem}

The genetic algorithm is an optimization method derived from the processes of creatures' evolution [12]. This is done by the creation of a population of individuals which their features are specified by chromosomes, selection the best individuals based on their fitness, and producing new population by cross-over, mutation and elitism operations. The ultimate aim of method is to find the maximum or minimum of an objective function.

The first step to solve our optimization problem is to define a proper objective function. Given that in many applications the purpose is to minimize the switching losses as well as the harmonic contents, the objective function can be chosen as follows

$$
O F=f\left(S w_{n}, H_{c}\right)
$$

where $S w_{n}$ is the switching number, which itself is proportional to the switching losses. Considering the fact that the output filter is a low-pass LC filter which effectively suppress the high frequency noises, the aim is to minimize the low-order harmonic contents in the output waveforms which are more difficult to filter out. Consequently, we define $H_{c}$ as

$$
H_{c}=g\left(v_{5}, v_{7}, W T H D\right)
$$

in which $v_{i}$ is the magnitude of $i$-th harmonic component, and WTHD is defined as

$$
W T H D=\frac{\sqrt{\sum_{i=2}^{n}\left(\frac{v_{i}}{i}\right)^{2}}}{v_{1}}
$$

The proposed chromosome consist of 2 sections. The first section expresses the sequence of duty cycles. In general

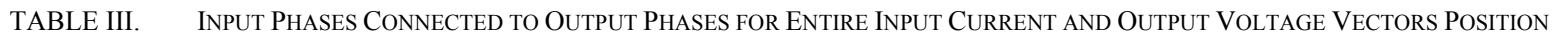

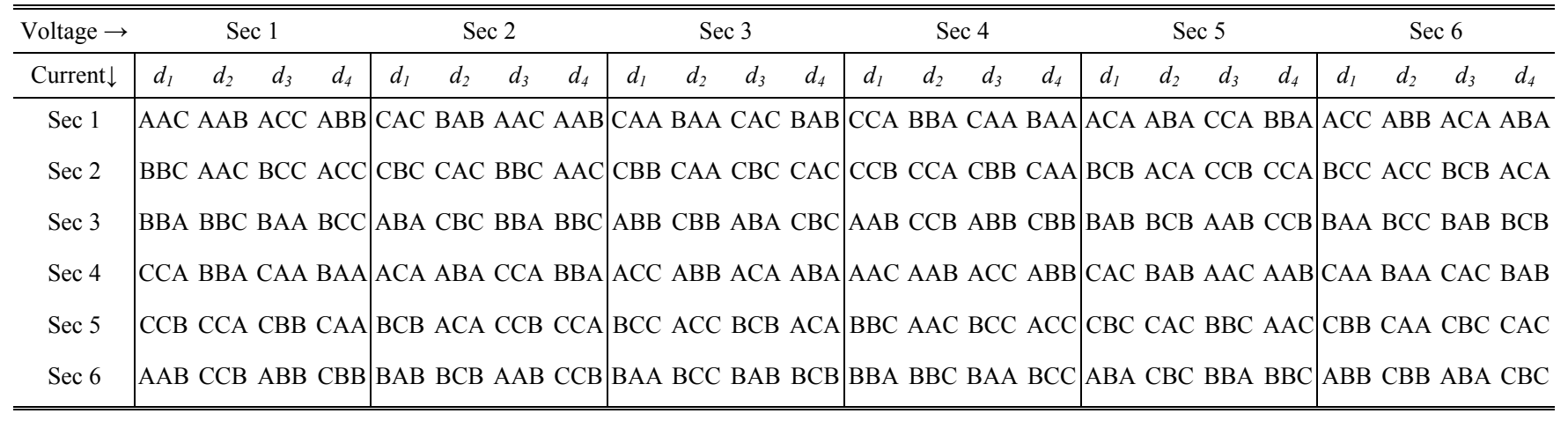




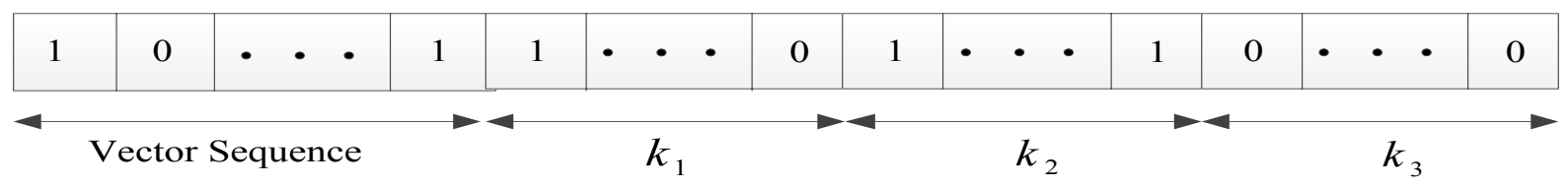

Figure 4. The chromosome structure.

state, 4 active duty cycles and 3 zero duty cycles exist in a period of switching. Taking into account that $7 !=5040$ different states will result from all possible combination of duty cycles, we represent them with 13 binary bits $\left(2^{13}=8192\right)$. Due to the probable nature of the genetic algorithm operators (cross-over and mutation), some generated binary codes may be out of range (5041 to 8192). To avoid these states, the invalid binary codes are scaled down by a factor $K$, and finally the results will be rounded. The scaling is defined as

$$
A=\left\{\begin{array}{l}
A \quad \text { if } A \leq 5040 \\
A \times K \quad \text { if } \quad 5040<A \leq 8192
\end{array}\right.
$$

in which $A$ is the decimal representation of binary bits, and $K$ is

$$
K=\frac{5040}{8192} .
$$

Another section of the chromosome specify the share of each zero duty cycle in the inactive portion of the switching period. These shares must be real variables smaller than one. Depending on the desired resolution of zero duty cycle's share of inactive time, the number of bits can change. On the other hand, the contribution of all three zero duty cycles to inactive time $\left(k_{1}, k_{2}\right.$, and $\left.k_{3}\right)$ must satisfy the following constraint

$$
k_{1}+k_{2}+k_{3}=1 \text {. }
$$

To meet this equality constraint implicitly, the share of zero duty cycles will be normalized as follows

$$
\begin{aligned}
& A_{1}=\frac{k_{1}}{k_{1}+k_{2}+k_{3}} \\
& A_{2}=\frac{k_{2}}{k_{1}+k_{2}+k_{3}} \\
& A_{3}=\frac{k_{3}}{k_{1}+k_{2}+k_{3}}
\end{aligned}
$$

where $A_{1}, A_{2}$ and $A_{3}$ are the new share of three zero duty cycles of whole the inactive time. Fig. 4 depicts the structure of the chromosome. It must be mentioned that the doublesided approach have been employed to increase the waveform symmetry and reduce the THD.

To complete the structure of the chromosome, consider table III. This table illustrates the input phases connected to the output phases for all possible combinations of input current and output voltage vectors. Having examined the table, one can find that there are only 18 unique states among 36 available combinations. As a result, the ultimate structure of the chromosome consists of 18 rows similar to Fig. 4, each row specifies the SVM switching pattern in that certain state.

\section{SimUlations AND DisCUSSION}

The matrix converter is simulated in Matlab/Simulink, and the adopted genetic algorithm has been applied to an objective function as follows

$$
O F=\frac{1}{\left(H_{c} / 10\right)^{4} \times(\text { Switching Numbers /3000 })^{2}}
$$

in which

$$
H_{c}=500 W T H D+1000 v_{5}+1000 v_{7}+200 v_{D C}
$$

The primary objective of this function is to decrease the harmonic contents of the output voltage, and the second target is to minimize to number of switchings. It must be noted that according to the application requirements, the weights of different terms in objective function can be changed.

The final chromosome obtained from the optimization is shown in table IV. To clarify the results, the sequence of duty cycles, obtained from the first section of chromosome, have been presented in table IV. In this table, $S_{i}$ shows the switching configuration of $i$-th state in the switching pattern.

To compare the performance of the proposed switching pattern with a conventional one, the 8 -switch method have been chosen. Both of them have been simulated in Matlab/Simulink. The simulation parameters are shown in table $\mathrm{V}$ and the output voltage harmonic components of two methods have been summarized in table VI. The number of switchings of two SVM methods during a fundamental input period is also reported. Clearly, the amplitude of low order harmonics, such as 5th and 7th are reduced, and the WTHD is improved. As mentioned before, the objective function of (24) has been weighted such that the WTHD and low harmonics have the primary importance in comparison with the number of switchings; consequently, the total number of switchings is higher than the conventional SVM. By adjusting the weights of (24), according to the application requirements, one can reach a satisfactory trade-off between the switching numbers and the harmonic contents.

TABLE IV. SIMULATION PARAMETERS

\begin{tabular}{cc}
\hline \hline R-L load & $\mathrm{R}=2[\Omega], \mathrm{L}=3.7[\mathrm{mH}]$ \\
Voltage Transfer Ratio (q) & 0.86 \\
Input Voltage (Peak amplitude) & $100[\mathrm{~V}]$ \\
Input Frequency & $50[\mathrm{~Hz}]$ \\
Output Frequency & $200[\mathrm{~Hz}]$ \\
Switching Frequency & $10000[\mathrm{~Hz}]$ \\
\hline \hline
\end{tabular}


TABLE V. FinAl Chromosome

\begin{tabular}{cccccccccc}
\hline \hline $\mathrm{S}_{1}$ & $\mathrm{~S}_{2}$ & $\mathrm{~S}_{3}$ & $\mathrm{~S}_{4}$ & $\mathrm{~S}_{5}$ & $\mathrm{~S}_{6}$ & $\mathrm{~S}_{7}$ & $d_{01}$ & $d_{02}$ & $d_{03}$ \\
\hline 1 & $0_{3}$ & 7 & $0_{2}$ & $0_{1}$ & 9 & 3 & 1 & 0 & 0 \\
8 & $0_{3}$ & $0_{1}$ & 9 & 3 & $0_{2}$ & 2 & 1 & 0 & 0 \\
1 & $0_{1}$ & 7 & 8 & $0_{3}$ & $0_{2}$ & 2 & 0.5 & 0 & 0.5 \\
$0_{3}$ & 9 & 3 & $0_{1}$ & 1 & 7 & $0_{2}$ & 0 & 1 & 0 \\
$0_{2}$ & $0_{3}$ & $0_{1}$ & 2 & 8 & 9 & 3 & 0 & 1 & 0 \\
$0_{2}$ & 2 & 1 & 7 & 8 & $0_{3}$ & $0_{1}$ & 0 & 0.5 & 0.5 \\
6 & $0_{2}$ & $0_{1}$ & $0_{3}$ & 4 & 7 & 9 & 0 & 1 & 0 \\
$0_{2}$ & 8 & $0_{3}$ & 5 & 6 & $0_{1}$ & 9 & 0 & 0.5 & 0.5 \\
$0_{3}$ & $0_{1}$ & 4 & 7 & $0_{2}$ & 8 & 5 & 1 & 0 & 0 \\
4 & $0_{3}$ & 6 & 9 & $0_{1}$ & 7 & $0_{2}$ & 0 & 1 & 0 \\
$0_{1}$ & 9 & 5 & 8 & $0_{3}$ & 6 & $0_{2}$ & 1 & 0 & 0 \\
$0_{1}$ & 7 & $0_{2}$ & 4 & 5 & 8 & $0_{3}$ & 0 & 0 & 1 \\
4 & $0_{3}$ & 1 & $0_{2}$ & $0_{1}$ & 3 & 6 & 1 & 0 & 0 \\
$0_{1}$ & $0_{3}$ & 6 & 3 & 5 & 2 & $0_{2}$ & 0 & 0.5 & 0.5 \\
$0_{3}$ & 5 & 2 & 1 & 4 & $0_{2}$ & $0_{1}$ & 0.5 & 0 & 0.5 \\
$0_{1}$ & 3 & 6 & 4 & 1 & $0_{3}$ & $0_{2}$ & 0 & 1 & 0 \\
3 & $0_{3}$ & $0_{1}$ & 6 & 2 & 5 & $0_{2}$ & 0.5 & 0.5 & 0 \\
$0_{2}$ & $0_{3}$ & 2 & 5 & $0_{1}$ & 4 & 1 & 0 & 0 & 1 \\
\hline \hline
\end{tabular}

TABLE VI. HARMONIC COMPONENTS OF TWO SVM SWITCHING PATTERNS

\begin{tabular}{ccc}
\hline \hline Order & $\begin{array}{c}\text { Conventional } \\
\text { SVM(\%) }\end{array}$ & Optimized SVM(\%) \\
\hline DC & $\mathbf{0 . 0 2}$ & $\mathbf{0 . 0 2}$ \\
1 & 100 & 100 \\
$\mathbf{5}$ & $\mathbf{0 . 5 9}$ & $\mathbf{0 . 3 1}$ \\
$\mathbf{7}$ & $\mathbf{0 . 2 9}$ & $\mathbf{0 . 1 8}$ \\
11 & 0.23 & 0.15 \\
13 & 0.16 & 0.13 \\
17 & 0.16 & 0.06 \\
19 & 0.22 & 0.13 \\
23 & 0.19 & 0.14 \\
25 & 0.17 & 0.26 \\
29 & 0.06 & 0.22 \\
31 & 0.31 & 0.28 \\
35 & 0.07 & 0.11 \\
37 & 0.63 & 0.45 \\
41 & 0.03 & 0.22 \\
43 & 2.89 & 0.71 \\
47 & 0.13 & 5.24 \\
49 & 8.9 & 5.76 \\
53 & 0.13 & 5.35 \\
55 & 4.25 & 3.22 \\
WTHD & $\mathbf{0 . 2 4 5 9}$ & $\mathbf{0 . 2 1 3 2}$ \\
Switching Numbers & 1604 & 2374 \\
\hline \hline
\end{tabular}

\section{CONCLUSION}

The possibility of designing a suitable switching pattern for the matrix converter space vector modulation by optimization methods has been examined. Furthermore, a genetic algorithm has been adopted to solve the optimization problem. An objective function to minimize the switching numbers and the total harmonic distortion has been proposed. To confirm the performance of the proposed method, the obtained results from optimization have been compared with a conventional switching algorithm (8 switch approach) through simulations conducted in Matlab/ Simulink.

\section{REFERENCES}

[1] M. Venturini, "A new sine wave in sine wave out, conversion technique which eliminates reactive elements," in Proc. Powercon 7, 1980, pp. E3/1-E3/15.

[2] J. Rodriguez, M. Rivera, J.W. Kolar, P.W. Wheeler, "A review of control and modulation methods for matrix converters," Industrial Electronics, IEEE Transactions on, vol.59, no.1, Jan. 2012, pp. 58-70, doi: 10.1109/TIE.2011.2165310.

[3] L. Huber and D. Borojevic, "Space vector modulated three-phase to three-phase matrix converter with input power factor correction," IEEE Trans. Ind. Appl., vol. 31, no. 6, Nov. 1995, pp. 1234-1246.

[4] D. Casadei, G. Serra, A. Tani, and L. Zarri, "Matrix converter modulation strategies: A new general approach based on space-vector representation of the switch state," IEEE Trans. Ind. Electron., vol. 49, no. 2, Apr. 2002, pp. 370-381.

[5] K.B. Larsen, A.H. Jorgensen, L. Helle, F. Blaabjerg, "Analysis of symmetrical pulse width modulation strategies for matrix converters," Power Electronics Specialists Conference, vol.2, no., 2002 pp. 899904, doi: 10.1109/PSEC.2002.1022568.

[6] P. Nielsen, F. Blaabjerg, J.K. Pedersen "Space vector modulated matrix converter with minimized number of switchings and a feedforward compensation of input voltage unbalance" Proc. of PEDES 1996, pp. 833-839.

[7] C. Klumpner, P. Nielsen, I. Boldea, F. Blaabjerg, "A New Modulator for Matrix Converter allowing for the Reduction of Input Current Ripple" Proc. of OPTIM'OO, 2000, pp. 487-492.

[8] H.J. Cha, P.N Enjeti, "An approach to reduce common-mode voltage in matrix converter," Industry Applications, IEEE Transactions on , vol.39, no.4, July-Aug.2003, pp.1151-1159.

[9] L. Helle, S. Munk-Nielsen, "A novel loss reduced modulation strategy for matrix converters", Proc. of PESC'O1, pp. 1102-1 107.

[10] D. Casadei, G. Serra, A. Tani, L. Zarri, "A novel modulation strategy for matrix converters with reduced switching frequency based on output current sensing," Power Electronics Specialists Conference, 2004. PESC 04. 2004 IEEE 35th Annual, vol.3, no., June 2004, pp. 2373- 2379.

[11] R. L. Haupt and S. E. Haupt, "Practical Genetic Algorithms," New York:Wiley, 1998. 